\title{
Major flaws in "Double and triple burden of non-communicable diseases and its determinants among adults in Bangladesh: Evidence from a recent demographic and health survey"
}

\author{
Rakib Islam ${ }^{1}$ and John Oldroyd ${ }^{2}$ \\ ${ }^{1}$ Monash University \\ ${ }^{2}$ Australian Catholic University
}

September 24, 2021

We read with interest the aforementioned paper by Al-Zubayer et al. 2021 ${ }^{1}$ that investigated the "double" and "triple" burden of non-communicable diseases defined as any two or three of hypertension, diabetes and overweight/obesity, respectively. When the prevalence of individual condition was examined it was found that "Almost 56\% had hypertension, $23.4 \%$ had diabetes and $24.6 \%$ had overweight or obesity".

We think the authors have made errors in their analysis that has resulted in unreliable prevalence estimates of hypertension, diabetes and overweight/obesity, and casting doubt on their estimates of burden of non-communicable diseases. This will cause confusion in the scientific and wider community and at worst, inappropriate allocation of resources to address the artificially elevated burden of diseases. We have identified errors related to the calculation of mean blood pressure, definition of diabetes, and classification of overweight/obesity, as outlined below.

Al-Zubayer et al. 2021 state that "interviewers have measured the respondents' blood pressure and it was taken at the beginning, in the middle and at the end of each interview. The mean value of these measurements was documented as the final result to measure hypertension ${ }^{1}$ ". The Bangladesh Demographic and Health Survey (BDHS) survey did measure blood pressure three times, however, the report states that "The average of the second and third measurements was used to report respondents' blood pressure values" ${ }^{2}$. This is a standard practice in research and clinical practice to reduce the possibility of 'white coat' hypertension from the first reading. The classification of hypertension based on the mean of all three measurements may have resulted in higher means, and an overestimate of the prevalence of hypertension.

The authors also state that "If the fasting plasma glucose values were $>6.1 \mathrm{mmol} / \mathrm{L}(110 \mathrm{mg} / \mathrm{dL})$ and/or taking any medicine for diabetes, then the participant is considered to be diabetic otherwise normal." ${ }^{1}$ However, this is an incorrect definition of diabetes because it includes pre-diabetes . Fasting blood glucose in the range 6.1 to $6.9 \mathrm{mmol} / \mathrm{L}$ is the level for pre-diabetes, as recommended by the $\mathrm{WHO}^{3}$. It is also not the classification used in the BDHS survey report which states that "Individuals were considered as having raised blood glucose or diabetes if they had a fasting blood glucose (FBG) equivalent level of $7 \mathrm{mmol} / \mathrm{L}$ or above at the time of the survey or reported currently taking prescribed medication for their high blood glucose or diabetes" ${ }^{2}$, which is consistent with the WHO classification ${ }^{3}$. As a result of including prediabetes, the prevalence of 'diabetes' reported by the authors (23.4\%) was more than double that reported in the BDHS survey report $(10 \%)^{2}$.

Al-Zubayer et al. 2021 used the WHO global cut-off for overweight/obesity (BMI [?]25kg/m2). However, we think it would have been more appropriate to use Asian cut-offs (BMI [?]23kg/m2) as suggested by the WHO expert consultation ${ }^{4}$. This is due to the high risk of type 2 diabetes and cardiovascular disease in Asian people at lower BMIs than the WHO global cut-offs ${ }^{4}$. 
We believe these are important misinterpretations, which need to be addressed by the authors.

\section{References}

1. Al-Zubayer MA, Ahammed B, Sarder MA, et al. Double and triple burden of non-communicable diseases and its determinants among adults in Bangladesh: Evidence from a recent demographic and health survey.International Journal of Clinical Practice 2021:e14613.

2. National Institute of Population Research and Training (NIPORT) aI. Bangladesh Demographic and Health Survey 2017-18. Dhaka, Bangladesh, and Rockville, Maryland, USA: NIPORT and ICF. 2020

3. World Health Organization. Definition and diagnosis of diabetes mellitus and intermediate hyperglycaemia: report of a WHO/IDF consultation. 2006

4. WHO Expert Consultation. Appropriate body-mass index for Asian populations and its implications for policy and intervention strategies. The Lancet 2004;363:157-63. 